\title{
Improved quantification for non-transferrin-bound iron measurement using high-performance liquid chromatography by reducing iron contamination
}

\author{
KATSUNORI SASAKI ${ }^{1}$, KATSUYA IKUTA $^{2}$, HIROKI TANAKA ${ }^{1}$, TAKAAKI OHTAKE ${ }^{2}$, \\ YOSHIHIRO TORIMOTO ${ }^{3}$, MIKIHIRO FUJIYA ${ }^{2}$ and YUTAKA KOHGO ${ }^{2}$ \\ ${ }^{1}$ Department of Gastrointestinal Immunology and Regenerative Medicine; ${ }^{2}$ Division of Gastroenterology and \\ Hematology/Oncology, Department of Medicine, Asahikawa Medical University; ${ }^{3}$ Oncology Center, \\ Asahikawa Medical University Hospital, Asahikawa, Hokkaido 078-8510, Japan
}

Received March 4, 2011; Accepted June 23, 2011

DOI: $10.3892 / \mathrm{mmr} .2011 .518$

\begin{abstract}
Non-transferrin-bound iron (NTBI) refers to all forms of iron in the plasma that bind to ligands other than transferrin, and is considered to be a marker of iron toxicity. A variety of analytical approaches for measuring NTBI have been reported; however, a clinically relevant level of sensitivity has yet to be achieved. In addition, insufficient values of NTBI in some patients and healthy subjects have led to the assumption that there may be contamination of reagents with background iron. The present study re-evaluated the analytical procedures of the assay with regard to the potential points of iron contamination in each step. NTA and tris carbonatocobaltate (III) solutions were prepared with removal of iron contamination, and then quantification of NTBI was performed. As a result, the sensitivity of the high-performance liquid chromatography (HPLC)-based NTBI method was improved by the successful reduction of background iron contamination. Application of our modified method proved that NTBI was detected even in healthy volunteers, although the concentrations were extremely low; the average NTBI levels were $0.206 \pm 0.091 \mu \mathrm{M}$ (males, $\mathrm{n}=20$ ) and $0.212 \pm 0.095 \mu \mathrm{M}$ (females, $\mathrm{n}=16$ ). Thus, our modification of the NTBI assay may be clinically meaningful, and may contribute to the understanding of the clinical significance of relatively low, but elevated concentrations of NTBI in diseases other than typical iron overload.
\end{abstract}

Correspondence to: Dr Katsuya Ikuta, Division of Gastroenterology and Hematology/Oncology, Department of Medicine, Asahikawa Medical University, 2-1-1-1 Midorigaoka-Higashi, Asahikawa, Hokkaido 078-8510, Japan

E-mail:ikuta@asahikawa-med.ac.jp

Key words: non-transferrin-bound iron, iron contamination, high performance liquid chromatography, quantification

\section{Introduction}

Non-transferrin-bound iron (NTBI) refers to all forms of iron in the plasma that bind to ligands other than transferrin (Tf), and was first measured by Hershko et al in 1978 (1). NTBI is increased during iron overload, and is considered to be a marker of iron toxicity (2). In addition, NTBI is decreased to the detection limit by long-term therapies with iron chelators such as deferoxamine and deferasirox in thalassemic patients, and by venesection in hemochromatotic patients $(3,4)$. Therefore, the determination of NTBI is crucial for evaluating and monitoring the risk of iron toxicity (5).

A variety of analytical approaches for measuring NTBI have been reported (6-14). In certain methods, NTBI is initially mobilized and chelated by shuttle molecules such as nitrilotriacetic acid (NTA), followed by separation of the chelated iron from serum proteins using ultra-filters. The ultra-filtrate was then analyzed by atomic absorption spectrometry (AAS) $(6,7)$, the colorimetric reaction with bathofenanthroline (8) or highperformance liquid chromatography (HPLC) $(9,10)$. In other methods, NTBI was mobilized and detected in the same reaction mixture without separation by ultra-filtration. Bleomycin (11), iron-sensitive fluorescence probes such as calcein (12), fluorescein-labeled desferrioxamine (13) and fluorescencelabeled apo-transferrin (apo-Tf) (14) were reported to be used for the latter methods.

Among these different systems for measuring NTBI, the HPLC method is one of the most convenient methods that was developed initially and was widely used. Consequently, HPLC has been considered to be a 'gold standard method' for NTBI measurement. Moreover, HPLC is more sensitive than most methods, with the exception of AAS. However, even using the HPLC method, it has not been possible to fully quantify NTBI at concentrations lower than $0.47 \mu \mathrm{M}$, which was reported as the limit of detection (LOD) (9). In addition, various studies on NTBI values of healthy individuals described NTBI concentrations as being negative values. For example, the ranges of NTBI determined by the HPLC method in healthy volunteers were reported to be $-0.94 \pm 0.08$ (15) or $-0.30 \mu \mathrm{mol} / 1$ (-1.5 to $0.6 \mu \mathrm{mol} / \mathrm{l})$ (16). Furthermore, the ability to monitor 
the serial changes in NTBI after the introduction of effective iron chelators, which sometimes lead to NTBI levels below the sensitivity of the conventional HPLC method or may lead to apparent negative values, is gaining in significance. These negative values of NTBI in certain subjects have indicated that there may be contamination of reagents and apparatus used for the method with iron and other metals. To determine whether or not it would be possible to overcome this problem, we re-evaluated the analytical procedures of the HPLC-based NTBI assay method with regard to the points of potential iron contamination in each step of the assay.

In the present study, the sensitivity of the HPLC-based NTBI assay method was improved by successfully reducing background iron and contamination with other metals. We believe that our quantification of NTBI may provide more clinically meaningful data, and the lower absolute values of NTBI, which were not previously determined, may contribute to a greater understanding of the significance of low, but elevated concentrations of NTBI in diseases other than typical iron overload.

\section{Materials and methods}

Reagents. The following materials were obtained and used without further purification: distilled water for HPLC (Wako, Tokyo, Japan), HPLC-grade acetonitrile (Wako), 1 M morpholinopropanesulfonic acid (MOPS) (Dojindo, Tokyo, Japan), nitrilotriacetic acid (NTA) (Nacalai Tesque, Tokyo, Japan), and Chelex ${ }^{\mathrm{TM}}-100$ chelating resin (Bio-Rad, Hercules, CA, USA). Iron-free distilled water was obtained by treatment of 3 liters of distilled water with $30 \mathrm{~g}$ of Chelex-100. After incubation for $5 \mathrm{~min}$ at room temperature, the resin was removed by filtration using an Econo-Column (Bio-Rad) (13). The 1-propyl-2-methyl-3-hydroxy-4(1H)-pyridinone hydrochloride (CP22), which has a higher affinity for iron than NTA and is capable of removing iron from $\mathrm{Fe}(\mathrm{III})-[\mathrm{NTA}]_{2}$, which develops color after forming a complex with iron, was synthesized and qualified by Intellim Co. (Osaka, Japan) according to the method of Dobbin et al (17). Sample tubes and pipette tips were selected from metal-free materials certified by BMBio Co. (Tokyo, Japan).

Preparation of NTA solution. There was a variation in iron content, with five batches of NTA with concentrations ranging from 0.479 to $1.124 \mu \mathrm{M}$ in $80 \mathrm{mM}$ solutions. Therefore, the NTA reagent with the lowest iron content was selected for the preparation of the stock and working solutions. NTA trisodium was dissolved in iron-free distilled water. The NTA solution was treated with DIAION ion-exchange resin to eliminate the trace of iron from the solution, and the stock was neutralized. The levels of iron in these reagents were confirmed directly by the HPLC-based system as described below, to evaluate the iron contamination levels.

Preparation of the $\mathrm{Na}_{3}\left[\mathrm{Co}\left(\mathrm{CO}_{3}\right)_{3}\right] \cdot 3 \mathrm{H}_{2} \mathrm{O}$ solution. Triscarbonatocobaltate (III) trihydrate was prepared as previously described (9). Briefly, cobalt (II) nitrate hexahydrate (Wako) was dissolved in iron-free distilled water. To oxidize Co (II) to Co (III), hydrogen peroxide was added dropwise to the cobalt (II) nitrate hexahydrate solution. The oxidized cobalt solution was slowly added to a cold slurry of sodium bicarbonate/iron-free distilled water with continuous stirring. The olive-green precipitate was filtered using Stericup-HV (Millipore, Billerica, MA, USA) and washed with cold ironfree distilled water. The precipitate was then washed with a small amount of absolute ethanol and dried. The dry green powder of $\mathrm{Na}_{3}\left[\mathrm{Co}\left(\mathrm{CO}_{3}\right)_{3}\right] \cdot 3 \mathrm{H}_{2} \mathrm{O}$ was dissolved in $1 \mathrm{M}$ sodium bicarbonate. The mixture was stirred at room temperature for $2 \mathrm{~h}$. After filtration through a Stericup-HV, the filtrate was allowed to stand for 2 days at room temperature and filtered again to remove small amounts of precipitate. The resulting clear solution was stored at $4^{\circ} \mathrm{C}$. The determination of cobalt ion in the final stock solution was made by an inductively coupled plasma mass spectrometer method, and the concentration of cobalt ion was found to be $12.6 \mathrm{mM}$. In addition, the trace of iron in this solution was measured by electrothermal AAS and the concentration of iron was $0.179 \mu \mathrm{M}$. The working solution of $\mathrm{Na}_{3}\left[\mathrm{Co}\left(\mathrm{CO}_{3}\right)_{3}\right] \cdot 3 \mathrm{H}_{2} \mathrm{O}$ was prepared by diluting with $1 \mathrm{M}$ sodium bicarbonate, and the final concentration of cobalt ion was found to be $5.0 \mathrm{mM}$.

Quantification of NTBI. NTBI concentrations were measured as previously described (9). However, a number of modifications were performed to increase the sensitivity of quantification.

Serum samples had been maintained at $-20^{\circ} \mathrm{C}$ until the time of measurement, at which point they were thawed by incubation at $37^{\circ} \mathrm{C}$ until no solid particles were observed, and then chilled on ice. First, $50 \mu \mathrm{l}$ of the $5 \mathrm{mM}$ tris-carbonatocobaltate (III) trihydrate $\left(\mathrm{Na}_{3}\left[\mathrm{Co}\left(\mathrm{CO}_{3}\right)_{3}\right] \cdot 3 \mathrm{H}_{2} \mathrm{O}\right)$ solution was added to $450 \mu \mathrm{l}$ of serum, and incubated at $37^{\circ} \mathrm{C}$ for $60 \mathrm{~min}$ to block any unsaturated transferrin sites. The resulting mixed solution contained $500 \mu \mathrm{l}$. The mixture was separated into two aliquots of $225 \mu \mathrm{l}$. Then, $25 \mu \mathrm{l}$ of $800 \mathrm{mM}$ NTA solution was added to one of the aliquots to chelate the NTBI in the serum, which should be non-specifically and weakly bound to serum proteins such as albumin and citrate. Solvent $(25 \mu \mathrm{l})$ used for the preparation of NTA solution was added to the other tube. Both tubes were incubated for $30 \mathrm{~min}$ at room temperature to produce a ferric-nitrilotriacetate complex, $\mathrm{Fe}(\mathrm{III})-[\mathrm{NTA}]_{2}$. The $\mathrm{Fe}(\mathrm{III})-[\mathrm{NTA}]_{2}$ was then separated from serum proteins by spinning the serum mixture through an Amicon Ultra- $0.5 \mathrm{ml}$ $30 \mathrm{~K}$ (Ultracel-30K membrane) (Millipore) at 14,000 x $\mathrm{g}$ for $30 \mathrm{~min}$ at $20^{\circ} \mathrm{C}$. The ultrafiltrate was transferred to a polypropylene screw-top vial (Waters, Milford, MA, USA).

A total of $20 \mu \mathrm{l}$ of each ultra-filtrate was then directly injected into a metal-free HPLC system. This system utilized non-metallic polyether-ethyl ketone tubing throughout, and a 2796 BioSeparation module with a degasser and sample heater-cooler, with a 2998 Photodiode array detector (Waters). The analytical columns used were an OmniSpher $5 \mu \mathrm{m} \mathrm{C18}$, G100x3 mm, glass column with a ChromSep guard column SS 10x2 mm (Varian/Agilent Technologies, Santa Clara, CA, USA). The chromatographic conditions were: flow rate $0.8 \mathrm{ml} / \mathrm{min}$; the mobile phase was isocratic containing $20 \%$ acetonitrile and $3 \mathrm{mM} \mathrm{CP} 22$ in $5 \mathrm{mM}$ MOPS buffer at pH 7.0; and the complex was detected at the visible detection wavelength of $450 \mathrm{~nm}$. NTBI as $\mathrm{Fe}(\mathrm{III})-[\mathrm{NTA}]_{2}$ was fractionated on the column, and then immediately on-column derivatized with $\mathrm{CP} 22$ to form a $\mathrm{Fe}(\mathrm{III})-[\mathrm{CP} 22]_{3}$ complex. The resulting orange-colored $\mathrm{Fe}(\mathrm{III})-[\mathrm{CP} 22]_{3}$ products were detected at 


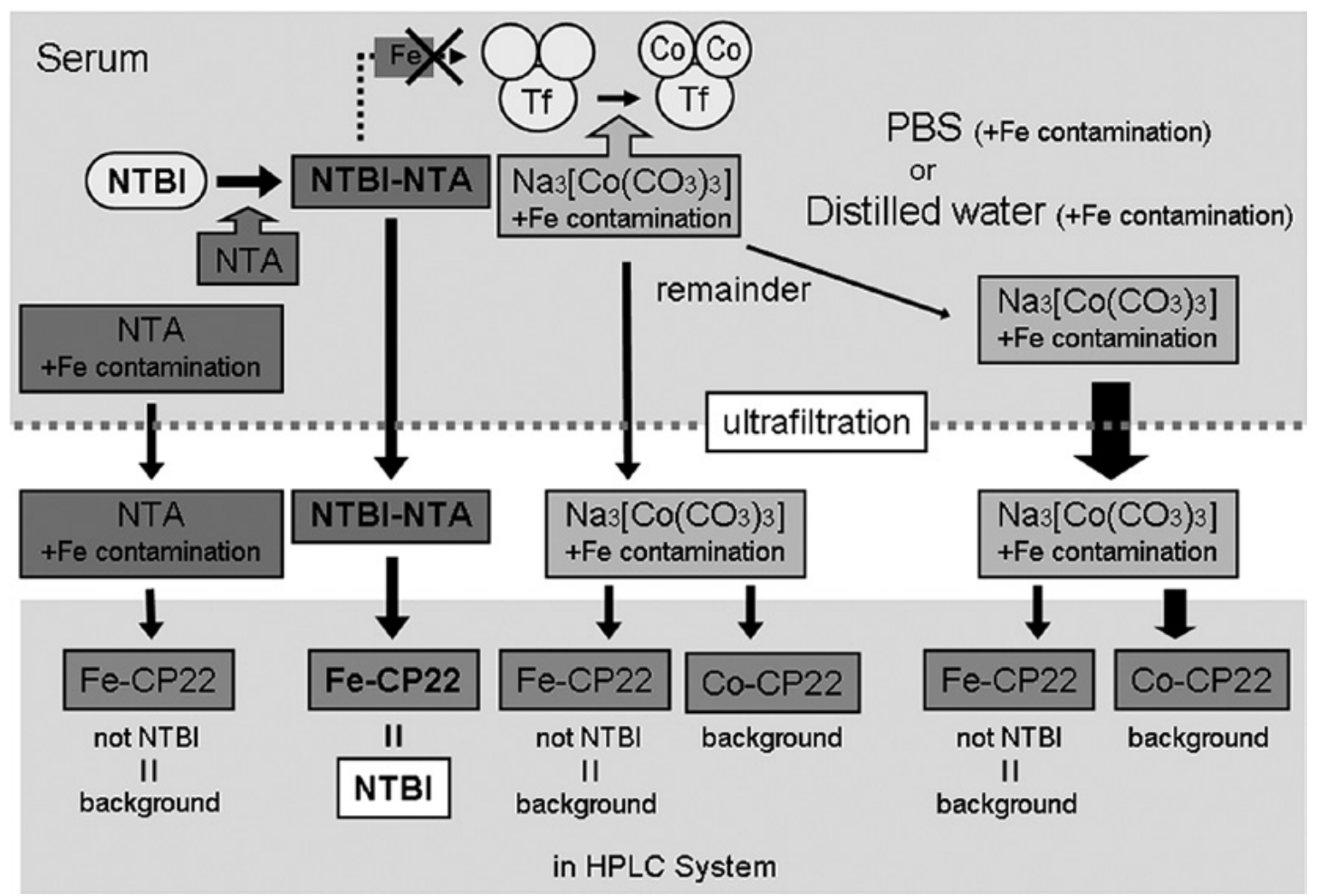

Figure 1. A schematic diagram of the NTBI analysis and the effect of heavy metal contamination. NTBI in serum was chelated by NTA and ultrafiltered. Unsaturated iron binding sites of apo-Tf in serum were previously blocked by $\mathrm{Na}_{3}\left[\mathrm{Co}\left(\mathrm{CO}_{3}\right)_{3}\right] \cdot 3 \mathrm{H}_{2} \mathrm{O}$, since the removal of iron from NTBI-NTA to apo-Tf should be prevented. Ultrafiltered NTBI-NTA transferred iron to another chelator, CP22, and then the level of iron was quantitated by a HPLC system. All of the reagents used were contaminated by iron, even in the NTA and $\mathrm{Na}_{3}\left[\mathrm{Co}\left(\mathrm{CO}_{3}\right)_{3}\right] \cdot 3 \mathrm{H}_{2} \mathrm{O}$ solutions. Distilled water was also contaminated with iron, which was eliminated by treatment with Chelex-100. In addition, the remainder of the $\mathrm{Na}_{3}\left[\mathrm{Co}_{(}\left(\mathrm{CO}_{3}\right)_{3}\right] \cdot 3 \mathrm{H}_{2} \mathrm{O}$ that was not used for occupation of unsaturated iron binding sites of apo-Tf was measured as background indistinguishable from pure NTBI. Thus, the contamination of iron in $\mathrm{Na}_{3}\left[\mathrm{Co}\left(\mathrm{CO}_{3}\right)_{3}\right] \cdot 3 \mathrm{H}_{2} \mathrm{O}$ solution led to an increase in the background, resulting in a decrease in the final NTBI value which was occasionally negative in previous studies.

$450 \mathrm{~nm}$. The iron concentration was derived from the calibration curve. Finally, the measured NTBI value of the tube without NTA was subtracted from that containing NTA. By this subtraction, the contamination of iron in the solvent used for the NTA solution should be offset.

Iron standard curve. Iron standards of concentrations ranging from 0-10 $\mu \mathrm{M}$ were prepared from a stock solution of $\mathrm{Fe}(\mathrm{III})$ $[\mathrm{NTA}]_{2}$ complex. Equal volumes of $160 \mathrm{mM}$ NTA and $20 \mathrm{mM}$ ferric chloride were mixed, and the mixture was titrated to pH 7-8 with $\mathrm{NaHCO}_{3}$. The determination of iron ion in the final stock solution was performed by electrothermal AAS. Dilutions were performed using iron-free distilled water treated with Chelex-100 resin. This iron-free distilled water was also used as the blank. Quantities of $20 \mu \mathrm{l}$ of each iron standard and blank were injected into the HPLC system described above. The measured iron value of the blank was subtracted from that containing $\mathrm{Fe}(\mathrm{III})-[\mathrm{NTA}]_{2}$ standards.

Application of the NTBI assay to serum samples from healthy volunteers. Blood samples were obtained with written permission from 36 healthy volunteers $(20$ males and 16 females) who did not have any obvious disease. The mean of ages and $\mathrm{Hb}$ levels of the subjects were 33.4 years (range 22-50) and $15.6 \mathrm{~g} / \mathrm{dl}$ (range 14.2-17.4) in males, and 33.8 years (range $22-44$ ) and $13.2 \mathrm{~g} / \mathrm{dl}$ (range 10.3-15.0) in females, respectively.

\section{Results}

NTBI analysis and the effect of heavy metal contamination. We hypothesized that the low sensitivities of the various NTBI quantification methods indicated the presence of iron or cobalt contamination in the assay system, which may affect the final NTBI values (Fig. 1).

Removal of iron contamination from reagent. We preliminarily observed that certain heavy metals, including iron, were contaminating the reagents used at many steps, including the iron chelating reagents (data not shown). Therefore, we attempted to remove the iron or cobalt contamination using various polyamine- or ion-exchange resins in addition to Chelex-100. The reduction of contaminated iron was evaluated by comparing iron levels determined prior to and after the addition of the chelating agents (Table I). The iron contamination in certain solvents including distilled water was completely removed by treatment with Chelex-100 resin.

Since the concentration of iron in NTA was not certified by the manufacturer, different batches may have different inherent iron contents. The concentration of iron in the untreated $80 \mathrm{mM}$ NTA solution was $87.00 \mu \mathrm{M}$, which was confirmed directly by HPLC-based quantification of NTBI as described above. On the other hand, the concentration of iron in Chelex-treated NTA solution was $51.76 \mu \mathrm{M}$. Subsequently, only $40 \%$ of iron contamination in the NTA 
Table I. Removal of iron contamination from the reagents.

\begin{tabular}{|c|c|c|c|c|}
\hline Reagents/solvents & $\begin{array}{c}\text { Residual heavy metal } \\
\text { contamination }\end{array}$ & Treatments & Assays & Results \\
\hline Distilled water & $\mathrm{Fe}$ & Chelex-100 & HPLC-CP22 & Not detected \\
\hline \multirow[t]{4}{*}{ NTA } & $\mathrm{Fe}$ & Chelex-100 & HPLC-CP22 & $\begin{array}{l}\text { Removal of } 40.5 \% \text { of } \\
\text { the contaminating } \\
\mathrm{Fe}\end{array}$ \\
\hline & & $\begin{array}{l}\text { The chelating resin that } \\
\text { has polyamine group } \\
\text { as chelating ligand }\end{array}$ & HPLC-CP22 & $\begin{array}{l}\text { Removal of } 78.5 \% \text { of } \\
\text { the contaminating } \\
\mathrm{Fe}\end{array}$ \\
\hline & & Ion-exchange resin & HPLC-CP22 & $\begin{array}{l}\text { Removal of } 99.8 \% \text { of } \\
\text { the contaminating } \\
\mathrm{Fe}\end{array}$ \\
\hline & & Selection of batch & HPLC-CP22 & $\begin{array}{l}\text { Reduction of the } \mathrm{Fe} \\
\text { contamination }\end{array}$ \\
\hline Tris-carbonatocobaltate (III) & $\mathrm{Fe}$ & Selection of batch & AAS & $\begin{array}{l}\text { Reduction of the } \mathrm{Fe} \\
\text { contamination }\end{array}$ \\
\hline
\end{tabular}

The iron contamination levels varied among the batches of NTA and cobalt (II) nitrate hexahydrate [used for the preparation of triscarbonatocobaltate(III)] reagents; therefore, batches with a low iron content were selected for further use. After eliminating the trace of iron in the NTA solutions, the removal ratio of iron was evaluated by comparing the iron levels determined before and after adding chelating agents. The concentrations of iron in untreated and treated NTA solutions were confirmed directly by HPLC-based quantification of NTBI as described in Materials and methods. The trace of iron in the tris-carbonatocobaltate (III) solution was measured by AAS.

solution was removed by treatment with Chelex-100 resin for $1 \mathrm{~h}$ at room temperature. The concentration of iron present in the polyamine-chelating resin-treated and the ion-exchange resin-treated $80 \mathrm{mM}$ NTA solution was $18.64 \mu \mathrm{M}$ and $0.10 \mu \mathrm{M}$, respectively. Thus, 78 and $99 \%$, respectively, of the iron contaminating the NTA solution was therefore removed.
Using the same treatment with polyamine-chelating resin against a different batch of $80 \mathrm{mM}$ NTA solution $(0.885 \mu \mathrm{M}$ iron), the concentration of iron was found to be $0.617 \mu \mathrm{M}$ (30.3\% removal of iron). On the other hand, treatment of the $80 \mathrm{mM}$ NTA solution with ion-exchange resin (0.885 $\mu \mathrm{M}$ iron), the iron that was identified as the NTBI peak in the HPLCbased quantification system was not be detected. Notably, we

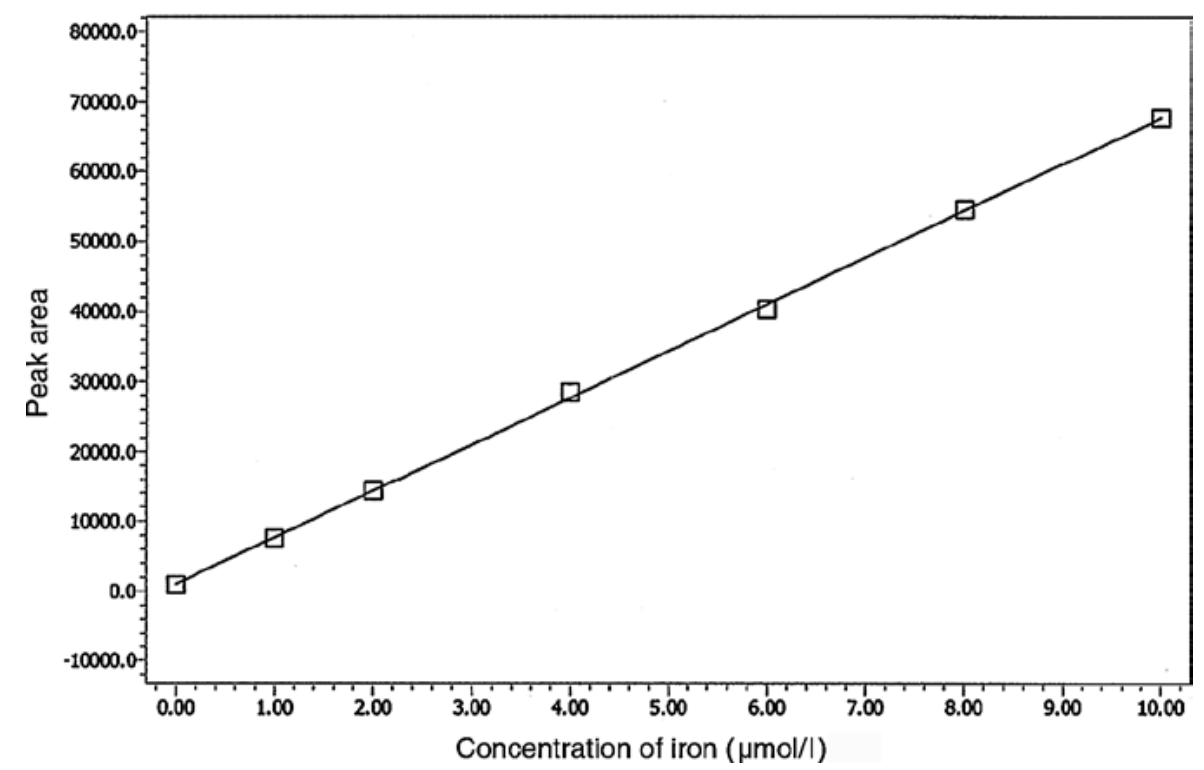

Figure 2. A series of Fe(III)-[NTA $]_{2}$ solutions with iron concentrations ranging from 0 to $10 \mu \mathrm{M}$ were prepared as the iron standards. Quantities of $20 \mu 1$ of each iron standard and blank were injected into the HPLC system. The measured iron value of the blank was subtracted from that containing Fe(III)-[NTA $]_{2}$ standards. 


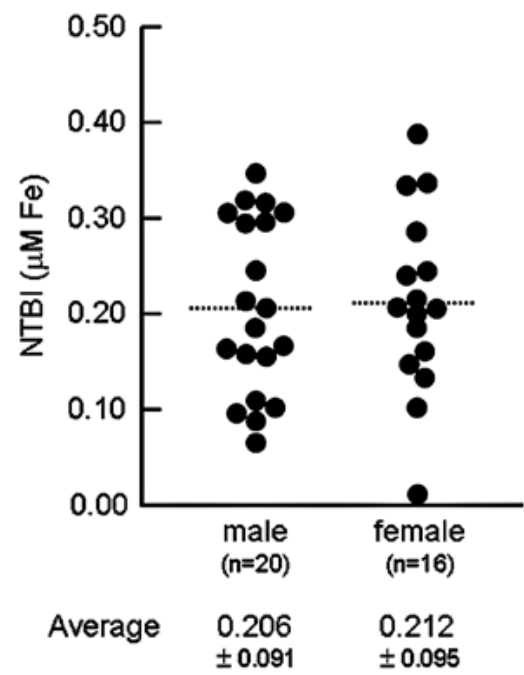

Figure 3. The serum NTBI in healthy volunteers $(n=36)$. No negative values were found in any of the serum samples from healthy volunteers. The average NTBI as $0.206 \pm 0.091 \mu \mathrm{M}$ (males, $\mathrm{n}=20)$ and $0.212 \pm 0.095 \mu \mathrm{M}$ (females, $\mathrm{n}=16$ ).

were able to reproducibly remove more than $95 \%$ of the iron contamination from the NTA solution. Therefore, treatment of the NTA solution using the ion-exchange resin was effective in minimizing the iron contamination of NTA.

However, one problem could not be resolved using chelating agents. In the present assay, tris-carbonatocobaltate (III) trihydrate was used for the previous saturation of apo-Tf prior to the addition of NTA, since displacement of iron from the NTBI-NTA complex to apo-Tf should be prevented. The remaining tris-carbonatocobaltate (III) trihydrate that was not used to occupy unsaturated sites of apo-Tf required ultrafiltration, since it was indistinguishable from NTBI-NTA. Ultra-filtered cobalt is chelated by CP22 in the same manner as NTBI-NTA, and can thus affect the NTBI values.

Therefore, we attempted to apply the subtraction method to offset the contamination of iron in the NTA solution and to determine the affect of the remaining cobalt on the quantification of NTBI. After the addition of tris-carbonatocobaltate (III) trihydrate solution, samples were separated in two tubes. The NTA solution was added to one of these, while solvent used for NTBI was added to the other tube. The measured NTBI value of the tube without NTA was subtracted from that of the tube containing NTA, offsetting the effect of the remaining cobalt that was not used to occupy the unsaturated Tf binding sites. This subtraction would also offset the contamination of iron in the solvent used for dissolving NTA and that in the tris-carbonatocobaltate (III) trihydrate solution, although the levels of iron contamination in these reagents appeared to be low after using the chelating agents.

Quantification of NTBI by standard curve. A standard curve for iron measured at $450 \mathrm{~nm}$, which was determined from an $\mathrm{Fe}(\mathrm{III})-[\mathrm{NTA}]_{2}$ solution at a range of 0 to $10 \mu \mathrm{M}$ is shown in Fig. 2. This standard curve appeared to be almost linear under the iron concentrations that were between 0 and $10 \mu \mathrm{M}$. Therefore, this standard curve was used to quantify NTBI in serum samples.
By applying our modified method, NTBI was detected even in healthy volunteers, although the concentrations were extremely low; the average NTBI levels were $0.206 \pm 0.091 \mu \mathrm{M}$ (males, $\mathrm{n}=20$ ) and $0.212 \pm 0.095 \mu \mathrm{M}$ (females, $\mathrm{n}=16$ ) (Fig. 3). No negative NTBI value was found even in healthy volunteers; which was possibly due to the fact that our subtraction method increased the sensitivity of quantification, since negative values of NTBI had often been observed previously, and had been presumed to be due to iron contamination in the reagents.

\section{Discussion}

NTBI has been reported to be toxic and may be involved in many iron-overload-related diseases. Under physiological conditions, iron should be shielded from reactions that lead to the formation of various reactive oxygen species by binding to Tf (18). However, NTBI is often present in redox reactions and produces reactive oxygen species that are toxic to cells (3). In addition, Tf-bound iron is mainly taken up through its specific receptor, transferrin receptor 1 (TfR1). Subsequently, only cells that highly express TfR 1 can uptake this iron. In contrast, there is no known regulatory mechanism for NTBI uptake by cells, and it is believed that NTBI does not enter the cells selectively (2). As a result, NTBI may cause damage in various organs, including the liver, heart and pancreas, depending on the final location of deposition.

Quantification of NTBI may be clinically significant. The diagnosis of iron overload is now based on evaluation of the serum Tf saturation and ferritin. However, Tf saturation may not be a reliable index due to its variability and low sensitivity, since Tf production may be decreased in patients with liver dysfunction. The serum ferritin levels are also affected by inflammation in addition to iron status. Thus, various factors other than iron status should be taken into consideration when using these values in the clinic. NTBI may be useful for differentiating abnormalities in iron metabolism from other pathologies, as NTBI should be increased in iron overloaded conditions, and may be a marker of iron overload even at the early stage. Moreover, NTBI levels can be utilized to monitor the status of patients with iron overload during treatment, since according to certain studies NTBI levels are decreased by long-term therapies with iron chelators in thalassemic patients and by venesection in hemochromatotic patients $(3,4)$.

The quantification of NTBI has been a challenging process. However, various methods have been reported (19). As sensitivities have been improving, it was noted that NTBI is likely to exist even under physiological conditions when Tf is not fully saturated $(5,20)$. This observation suggests that NTBI was not merely excess iron above the iron binding capacity of Tf. However, the sensitivities of the NTBI quantification methods used in the past have not been sufficiently high. As a result, low concentrations of NTBI could not be precisely determined. Therefore, little information is available with regard to the NTBI in subjects not in severe iron overload or in healthy individuals.

A variety of analytical approaches for measuring NTBI have been reported. Their principles and practical applications differ markedly. In certain methods, NTBI was initially 
mobilized and chelated by shuttle molecules including NTA, followed by separation of the chelated iron from serum proteins using ultra-filters with a molecular weight cutoff of 30,000 , after which the ultra-filtrate was analyzed by AAS $(6,7)$, a colorimetric reaction with bathofenanthroline (8) or HPLC $(9,10)$. In other methods, NTBI was mobilized and detected in the same reaction mixture without separation by ultra-filtration. Bleomycin (11), iron-sensitive fluorescence probes such as calcein (12), fluorescein-labeled deferoxamine (13) and fluorescence-labeled apo-Tf (14) were reported to be used for the latter methods. By using these methods, labile plasma iron (LPI), which has been considered to be the highly redox-active iron fraction in NTBI, could be measured.

Among these different systems for measuring NTBI, the HPLC method is a well-established method that has high sensitivity for NTBI detection with high specificity and reproducibility. However, even the HPLC method did not completely quantify NTBI, especially at low concentrations. For example, several studies on the NTBI values of healthy individuals described NTBI concentrations as negative values $(15,16)$. Therefore, improvement in the sensitivity of NTBI detection is required to determine the usefulness of NTBI quantification in the clinic. However, iron contamination posed a serious problem in achieving this high sensitivity for NTBI quantification, as iron contamination may be present in various reagents and in the equipment and instruments, as well as in the laboratory environment.

In conclusion, we established a reliable, stable, reproducible NTBI quantification method utilizing an HPLC system with high sensitivity, mainly by the successful removal of contaminated iron in the reagents used for the assay. Results of our improved method showed no negative NTBI values, even in healthy volunteers, indicating that the evaluation of NTBI and comparison of the levels in affected subjects to those in healthy individuals is now feasible. Subsequently, the clinical significance of NTBI measurements in various disease conditions and normal states can be elucidated. Moreover, this improved method for NTBI quantification may contribute to the future research concerning NTBI, as this modified method allows for the more sensitive detection of NTBI, and may provide a widely acceptable marker for evaluating and monitoring of iron metabolism and iron toxicity.

\section{References}

1. Hershko C, Graham G, Bates GW and Rachmilewitz EA: Non-specific serum iron in thalassaemia: an abnormal serum iron fraction of potential toxicity. Br J Haematol 40: 255-263, 1978.

2. Hershko C: Mechanism of iron toxicity. Food Nutr Bull 28: S500-S509, 2007.
3. Hershko C: Pathogenesis and management of iron toxicity in thalassemia. Ann N Y Acad Sci 1202: 1-9, 2010.

4. Meo A, Ruggeri A, La Rosa MA, Zanghi L, Morabito N and Duca L: Iron burden and liver fibrosis decrease during a long-term phlebotomy program and iron chelating treatment after bone marrow transplantation. Hemoglobin 30: 131-137, 2006.

5. Breuer W, Hershko C and Cabantchik ZI: The importance of non-transferrin bound iron in disorders of iron metabolism. Transfus Sci 23: 185-192, 2000.

6. Dürken M, Nielsen P, Knobel S, et al: Nontransferrin-bound iron in serum of patients receiving bone marrow transplants. Free Radic Biol Med 22: 1159-1163, 1997.

7. Jakeman A, Thompson T, McHattie J and Lehotay DC: Sensitive method for nontransferrin-bound iron quantification by graphite furnace atomic absorption spectrometry. Clin Biochem 34: 43-47, 2001.

8. Zhang D, Okada S, Kawabata T and Yasuda T: An improved simple colorimetric method for quantitation of non-transferrinbound iron in serum. Biochem Mol Biol Int 35: 635-641, 1995.

9. Gosriwatana I, Loreal O, Lu S, Brissot P, Porter J and Hider RC: Quantification of non-transferrin-bound iron in the presence of unsaturated transferrin. Anal Biochem 273: 212-220, 1999.

10. Loreal O, Gosriwatana I, Guyader D, Porter J, Brissot P and Hider RC: Determination of non-transferrin-bound iron in genetic hemochromatosis using a new HPLC-based method. J Hepatol 32: 727-733, 2000.

11. Gutteridge JM and Hou YY: Iron complexes and their reactivity in the bleomycin assay for radical-promoting loosely-bound iron. Free Radic Res Commun 2: 143-151, 1986.

12. Breuer W, Ronson A, Slotki IN, Abramov A, Hershko C and Cabantchik ZI: The assessment of serum nontransferrin-bound iron in chelation therapy and iron supplementation. Blood 95: 2975-2982, 2000.

13. Breuer W, Ermers MJ, Pootrakul P, Abramov A, Hershko C and Cabantchik ZI: Deferrioxamine-chelatable iron, a component of serum non-transferrin-bound iron, used for assessing chelation therapy. Blood 97: 792-798, 2001.

14. Breuer W and Cabantchik ZI: A fluorescence-based one-step assay for serum non-transferrin-bound iron. Anal Biochem 299: 194-202, 2001.

15. Porter JB, Abeysinghe RD, Marshall L, Hider RC and Singh S: Kinetics of removal and reappearance of non-transferrin-bound plasma iron with deferoxamine therapy. Blood 88: 705-713, 1996.

16. De Valk B, Addicks MA, Gosriwatana I, Lu S, Hider RC and Marx JJ: Non-transferrin-bound iron is present in serum of hereditary haemochromatosis heterozygotes. Eur J Clin Invest 30: 248-251, 2000.

17. Dobbin PS, Hider RC, Hall AD, et al: Synthesis, physicochemical properties, and biological evaluation of N-substituted 2-alkyl3-hydroxy-4 $(1 \mathrm{H})$-pyridinones: orally active iron chelators with clinicalpotential. J Med Chem 36: 2448-2458, 1993.

18. Aisen P, Enns C and Wessling-Resnick M: Chemistry and biology of eukaryotic iron metabolism. Int J Biochem Cell Biol 33: 940-959, 2001.

19. Jacobs EM, Hendriks JC, van Tits BL, et al: Results of an international round robin for the quantification of serum nontransferrin-bound iron: need for defining standardization and a clinically relevant isoform. Anal Biochem 341: 241-250, 2005.

20. Bradley SJ, Gosriwitana I, Srichairatanakool S, Hider RC and Porter JB: Non-transferrin-bound iron induced by myeloablative chemotherapy. Br J Haematol 99: 337-343, 1997. 